\title{
Multiple Symbol Differential Detection of MPSK which is Invariant to Frequency Offset using Maximum-likelihood detection
}

\author{
Fahmida Tarannum ${ }^{1,}$ P. Govardhan ${ }^{2}$ \\ ${ }^{I}$ (M.Tech Student, Sree Chaitanya College of Engineering, Karimnagar, Andhra Pradesh, India) \\ ${ }_{2}^{2}$ (Associate Professor,ECE, Sree Chaitanya College of Engineering, Karimnagar, Andhra Pradesh, India)
}

\begin{abstract}
It is well known that classical differential detection of MPSK signals, wherein the information is encoded as the first order phase difference, is a simple and robust form of communication in environments not subject to frequency variation. For channels that introduce into the carrier a random frequency shift, eg., those associated with moving vehicles, classical differential detection as above may yield poor performance, particularly if the frequency shift is an appreciable fraction of the data rate. In such situations, one must resort to a form of differential detection that encodes the information as higher order (second order for constant frequency shift) phase difference process. It is shown that the proposed receiver is robust to the distortions caused by the random frequency variation. A lower bound on the error probability of the proposed MSDD receiver is also derived and compared to that of an autocorrelation demodulator for the case where the observation interval approaches infinity.
\end{abstract}

\section{Introduction}

Differential detection of phase-shift keying (PSK) signals is a well-known strategy for mitigating the performance degradation due to unknown phase offset. The constellation rotation caused by the phase offset can be removed using a differential PSK (DPSK) modulation scheme along with a differential detector. However, this detector suffers from a signal-to-noise power ratio (SNR) loss compared to a coherent detector. An effective means to mitigate this SNR loss is known as multiple-symbol differential detection (MSDD). The MSDD scheme is, indeed, a more general case of the conventional differential detection in which more than two consecutive samples are utilized to detect the information symbols. It is shown in that by increasing the number of received samples in MSDD, the receiver performance approaches that of coherent demodulation of DPSK signals. However, the MSDD receiver analysis assumes that the frequency offset equals zero. In the case of nonzero frequency offset, conventional MSDD must take the frequency offset into account. Otherwise, increasing the number of the received samples in MSDD degrades the performance very quickly. A double DPSK (DDPSK) modulation scheme (also referred to as second-order phase difference modulation) has been proposed for the case when the frequency offset is unknown. In the two types of demodulators for this modulation scheme have been introduced, the autocorrelation demodulator (ACD) and the optimum $I-Q$ demodulator. This reference also proposed an MSDD technique based on ACD for DDPSK signals and showed that this structure is frequency offset invariant. Nevertheless, even under the most optimistic conditions, i.e., an infinite number of received samples and vanishingly small noise power, the proposed receiver still requires $3 \mathrm{~dB}$ more SNR than coherent detection of differentially encoded PSK signals.

In this paper, we study the effect of frequency variations on a PSK signal transmitted over an additive white Gaussian noise (AWGN) channel. We show that frequency offset attenuates the amplitude of the transmitted signal and rotates its constellation points about the origin by a time-varying phase. Then, we derive a MSDD scheme to demodulate a DDPSK signal and show that this scheme is not sensitive to constellation rotation caused by the frequency offset. The proposed demodulator suffers from a SNR loss compared to a conventional MSDD with DPSK modulation when frequency offset is not present. However, as will be seen in the sequel, the SNR loss can be less than $3 \mathrm{~dB}$ for some modulation schemes resulting in a net performance gain relative to the ACD-based MSDD scheme proposed.

This paper is organized as follows. In Section II, we present the signal model for the cases where a rectangular pulse- shaping filter and a bandlimited pulse-shaping filter are used at the transmitter. In Section III, we propose a new MSDD scheme for DDPSK signals and derive a lower bound on its error probability. Numerical results are presented in Section IV. In Section V, some conclusions are drawn 
- Bandpass Received Signal

$---\cdot$ Demodulated Signal

- - - - Receiver Filter

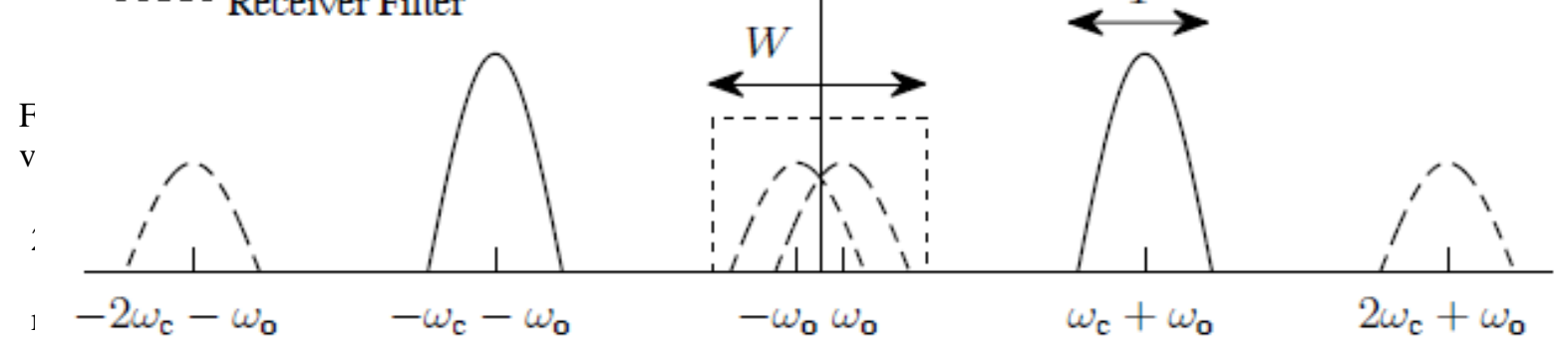

$$
r(t)=s_{k} e^{j\left(2 \pi f_{0} t+\bar{\phi}\right)}+n(t) \quad(k-1) T \leq t \leq k T
$$

where $s k$ is the $k$ th information symbol, $f_{0}$ and $\bar{\phi}$ are the frequency offset and phase offset, respectively, $T$ is the symbol interval and $n(t)$ is the baseband complex-valued AWGN process with mean zero and power spectral density $N_{0} /$. We assume that $s k$ is defined as

$$
s_{k}=\sqrt{\varepsilon_{s}} e^{j \theta_{k}}
$$

where $\mathcal{E}_{\mathrm{S}} \quad$ is the received energy per symbol, $\quad \theta k \in\{2 \pi m / M\}_{m=0}^{M-1}$ is the transmitted phase at the kth symbol interval and $M$ denotes size of the constellation. The received signal is passed through a correlation demodulator to obtain the decision statistic at the $k$ th symbol interval, normalized to $T$, as

$$
\begin{aligned}
& r_{k}=\int_{(k-1) T}^{k T}\left[s_{k} e^{j\left(2 \pi f_{0}+\bar{\phi}\right)}+n(t)\right] d t \\
& =s_{K}\left(\frac{\sin \left(\frac{\psi}{2}\right)}{\frac{\psi}{2}}\right) e^{j(k \psi+\phi)}+n_{K}
\end{aligned}
$$

Where $\psi=2 \pi f_{0} T, \phi=\bar{\phi}+\psi / 2$ and $n_{k}$ is an AWGN sample with mean zero and variance $\sigma_{n}^{2}=N_{0}$. The frequency offset has two adverse effects on the received signal. It rotates the constellation points about the origin with a radial speed of $\omega_{0}=2 \pi f_{0}$. It also decreases the signal amplitude by a factor of $\sin (\psi / 2) /(\psi / 2)$. Fortunately, for small values of frequency offset, the $\sin (\psi / 2) /(\psi / 2)$, term decays slowly with $\psi$, and for $|\psi|<\pi / 4$ the amplitude decay is less than $2.5 \%$. Therefore, we assume in the sequel that $\psi$ is small enough that we can approximate $\sin (\psi / 2) /(\psi / 2)$. with unity. Thus, (3) can be approximated as

$$
r_{k}=s_{k} e^{j(k \psi+\phi)}+n_{k}
$$

As we will see in the sequel, this assumption has little effect on the predicted performance of the proposed receiver provided that $|\psi|<\pi / 4$.

\subsection{Bandlimited Pulse-Shaping}

The Signal model presented in Section 2.1 is based on the premise that a rectangular pulse shaping filter is used at the transmitter. However, in practical systems the transmission channels have a limited bandwidth which distorts the rectangular pulse and causes intersymbol interference (ISI). In this section we describe how the signal model changes if the transmitter pulse-shaping filter, $p_{T}(t)$, is bandlimited. 
Fig. 1 shows the frequency domain representation of the bandlimited received signal in the presence of the frequency offset before and after demodulation. Normally, it is assumed that the receiver filter, $p_{R}(t)$, is matched to $p_{T}(t)$ maximize the output SNR. It is also assumed that the combination of $p_{T}(t)$ and $p_{R}(t)$ satisfies Nyquist's first criterion for zero intersymbol interference (ISI). In the presence of unknown frequency offset, this procedure will cause ISI because after demodulation the frequency offset changes the frequency response of the transmitter filter, $p_{T}(w)$, as seen in Fig.1. An alternative to this procedure has been proposed in which a root-raised cosine filter with $100 \%$ excess bandwidth is used at the transmitter along with a brick wall filter at the receiver, i.e.,

$$
\begin{aligned}
& p_{T}(w)=\left\{\begin{array}{l}
\sqrt{2 T} \cos \left(\frac{\omega T}{4}\right),|\omega| \begin{array}{l}
\leq \\
0
\end{array} \mid>\frac{2 \pi}{T} \\
0,
\end{array}\right. \\
& P_{R}(\omega)=\left\{\begin{array}{l}
\sqrt{\frac{T}{2}},|\omega| \leq W \\
0,
\end{array} \mid>W\right.
\end{aligned}
$$

Then, the kth decision statistic is formed by taking two samples of the filtered received signal at $\mathrm{t}=(\mathrm{k}+1 / 4) \mathrm{T}$ and $\mathrm{t}=(\mathrm{k}-1 / 4) \mathrm{T}$ and summing the resulting samples. It is shown that this procedure is ISI-free and does not degrade the SNR relative to the matched filter case provided that $p_{R}(t)$ and $p_{T}(t)$ have the same single-sided bandwidth $2 \pi / \mathrm{T}$.

Assume now that the bandwidth of $P_{R}(\omega), \mathrm{W}$, is large enough to ensure that $P_{T}(\omega)$ is undistorted. Then, the filtered received signal is given by

$$
r(t)=\sum_{l=-\infty}^{\infty} s_{l} p(t-I T) e^{j\left(\omega_{0} t+\phi\right)}+n(t)
$$

Where

$$
p(t)=\frac{4}{\pi}\left(\frac{\cos \left(\frac{2 \pi t}{T}\right)}{1-\left(\frac{4 t}{T}\right)^{2}}\right)
$$

And $\mathrm{n}(\mathrm{t})$ is a zero-mean Gaussian wide-sense stationary process with autocorrelation function

$$
R_{n}(\tau)=\frac{N_{0} W T}{4 \pi} \sin c\left(\frac{W \tau}{\pi}\right)
$$

The kth decision statistic is therefore given by

$$
\begin{aligned}
& r_{k}=r\left(k T-\frac{T}{4}\right)+r\left(k T+\frac{T}{4}\right) \\
& =\cos \left(\frac{\psi}{4}\right) s_{k} e^{j(k \psi+\phi)}+n_{+}+n_{-} \\
& n_{ \pm}=n\left(k T \pm \frac{T}{4}\right)
\end{aligned}
$$

Similar to the rectangular pulse-shaping case the amplitude of the signal decays by a factor of $\cos (\psi / 4)$. However, again, this decay is less than $2 \%$ for $|\psi|<\pi / 4$.

Another inherent problem with this approach is the noise enhancement caused by $P_{R}(\omega)$. Indeed in the presence of the frequency offset, the bandwidth of $P_{R}(\omega)$ is greater than $2 \pi / T$. As a result, the variances of $n_{+}$and $n_{-}$increase relative to the case where $W=2 \pi / T$. Interestingly, when $2 \pi / T<W<4 \pi / T$ the correlation 
between $\mathrm{n}_{+}$and $\mathrm{n}_{-}$is negative which reduces the variance of the overall noise. In order to quantify this fact, we assume that $\mathrm{W}=2 \pi(1+\alpha) / \mathrm{T}$ where $\alpha>0$. Then, the variance of the overall noise term (9a) can be evaluated as

$$
\sigma_{n}^{2}=N_{0}(1+\alpha)[1+\sin c(1+\alpha)]
$$

Where $\mathrm{N}_{0}(1+\alpha) \operatorname{sinc}(1+\alpha)$ denotes the contribution of the correlation between $\mathrm{n}_{+}$and $\mathrm{n}_{-}$. Now one can show that for $0 \leq \alpha \leq 0.125$, or equivalently, $|\psi| \leq \pi / 4, \sigma_{n}^{2}$ increases by less than $0.3 \%$ relative to the case where $\psi=0$. Hence, in the case of bandlimited signaling eq. (9a) can be still be well approximated by eq (4) provided that $|\psi| \leq \pi / 4$.

\subsection{Discussion}

As seen in sections 2.1 and 2.2, the amplitude loss due to frequency offset is negligible when $|\psi|<$ $\pi / 4$. In contrast, the constellation rotation caused by the frequency offset can degrade the performance rather rapidly even for small values of $\psi$. This is because the angle of rotation is a function of time and varies from one symbol interval to the other. The time-varying constellation rotation can be converted to a time-invariant rotation by using a DPSK modulation scheme, i.e.,

$$
\theta_{k} \square \sum_{i=1}^{k} \dot{\theta}_{i}
$$

Where $\dot{\theta}_{i}$ is the ith encoded information phase and $\dot{\theta}_{i}=\theta_{\mathrm{i}-} \theta_{\mathrm{i}-1}$. Assume that the term exp (jk $\left.\psi\right)$ in (4) is absorbed into $\mathrm{s}_{\mathrm{K}}$. Then, (4) can be written as

Where

$$
r_{K}=u_{k} e^{j \phi}+n_{K}
$$

$$
u_{k}=\sqrt{\varepsilon_{s}} \exp \left[j \sum_{i=1}^{K}\left(\dot{\theta}_{i}+\psi\right)\right]
$$

This means that in the presence of the frequency offset, the information phases should be chosen from $\{2 \pi m / M+\psi\}_{m=0}^{M-1}$ rather than $\{2 \pi m / M\}_{m=0}^{M-1}$. In other words, the effect of $\psi$ on a DPSK signal is similar to the effect of phase offset on a PSK signal. Therefore, a DDPSK modulation should be invariant to frequency offset as the DPSK modulation is invariant to phase offset.

\subsection{Receiver Derivation}

\section{Frequency Offset Insensitive Differential Detection}

Assume that the frequency offset and the phase offset are constant over N successive samples. Then, using (4) one can define a new variable $r_{k}$ as

$$
\begin{aligned}
& r_{k} \square r_{k} r_{k-1}^{*} \\
& =s_{k} s_{k-1}^{*} e^{j \psi}+n_{k}
\end{aligned}
$$

Where

$$
n \square s_{k} e^{j(k \psi+\phi)} n_{k-1}^{*}+n_{k} s_{k-1}^{*} e^{-j[(k-1) \psi+\phi]}+n_{k} n_{k-1}^{*}
$$

Assume that a PSK modulation is employed, i.e., $\mathrm{s}_{\mathrm{k}}$ is given by (2). Then, the first and second terms on the right of (13b) are independent Gaussian random variables each with mean zero and variance $\varepsilon_{s} \sigma_{n}^{2}$. In contrast, the last term has Complicated probability density function. However, for practical SNR values this term is relatively small compared to the first two and can be ignored. Moreover, it can be shown that 
$n_{k}$ and $n_{\text {l }}$ are uncorrelated for $\mathrm{k} \neq 1$. Therefore, we approximate $\left\{n_{k}\right\}$ as a zero-mean Gaussian random sequence with

$$
E\left\{n_{k} n_{\ell}\right\}=\varepsilon_{s} \sigma_{n}^{2} \delta(k-\ell)
$$

Where $\delta($.$) denotes the Kronecker delta function.$

Assume now that $\psi$ has a uniform distribution over the interval $[0,2 \pi)$ and that any amplitude variations due to frequency offset can be ignored. Also assume that in (13a) the term $s_{k} s_{k-1}^{*}=\varepsilon_{s} \exp \left(j \dot{\theta}_{k}\right)$ denotes the kth transmitted symbol. Then the maximum-likelihood (ML) receiver should maximize the metric

$$
\begin{aligned}
\Lambda & =\left|\sum_{i=0}^{N-1} r_{k-i} e^{-j \dot{\theta}_{k-i}}\right|^{2} \\
& =\left|\sum_{i=0}^{N-1} r_{k-i} e^{-j\left(\dot{\theta}_{k-i}-\dot{\theta}_{k-N+1}\right.}\right|^{2}
\end{aligned}
$$

Where the last equation follows from the fact that multiplying the argument of the $|$.$| in (14) by$ $\dot{\theta}_{k-N+1}$ does not change the metric [2]. ${ }^{1}$ Note that in this case we need N+1 samples to detect N-1 information symbols. It can be readily verified that

$$
\dot{\theta}_{k-i}-\dot{\theta}_{k-N+1}=\sum_{m=0}^{N-i-2} \ddot{\theta}_{k-i-m}
$$

where now $\ddot{\theta}=\dot{\theta}_{k}-\dot{\theta}_{k-1}$ is the information phase at the kth time interval. Thus, (15) can be rewritten as

$$
\Lambda=\left|r_{k-N+1}+\sum_{i=0}^{N-2} r_{k-i} \exp \left[-j \sum_{m=0}^{N-i-2} \ddot{\theta}_{k-i-m}\right]\right|^{2}
$$

Clearly, the metric in (17) is independent of the frequency offset and the phase offset. Assuming that $\theta_{-1}=\theta_{0}=0$ and using the identity $\theta_{k}=2 \theta_{k-1}-\theta_{k-2}+\ddot{\theta}_{k}$ one obtains

$$
\begin{aligned}
\theta_{k} & =\sum_{i=1}^{k}(k-i+1) \ddot{\theta} \\
& =\underbrace{\sum_{i=1}^{k} \overbrace{\sum_{m=1}^{i} \ddot{\theta}_{m}}^{\text {FirstDPSK }}}_{\text {SecondDPSK }}
\end{aligned}
$$

Encoding the information symbols using (18) is equivalent to encoding the information symbols using a DPSK encoder once, and then encoding the resulting symbols one more time with the same encoding rule. Note that the analysis presented in this section serves as the proof of optimality for the MSDD receiver when the $\left\{n_{k}\right\}$ are Gaussian. This occurs when the cross-noise term in (13b) is negligible, i.e., when the SNR is relatively large. 


\subsection{Error Probability Analysis}

For DDPSK signals, the I-Q demodulator is a special case of the proposed receiver when $\mathrm{N}=2$. In the absence of the frequency offset, an exact expression for the BER of the I-Q demodulator with binary DDPSK modulation has been derived. For higher order DDPSK signals, i.e., when $\mathrm{M}>2$, an exact expression for BER of the MSDD is not known to the best of author's knowledge. However, an upper bound for the symbol error rate (SER) for the case where $\mathrm{N}=2$ has been derived.

We now obtain a reasonably tight lower bound on the BER of the MSDD for the case where $N \rightarrow \infty$. To this end the performance of MSDD with DPSK modulation and $N \rightarrow \infty$, lower bounded by that of the coherent detection of differentially encoded PSK. i.e.,

$$
P_{s}^{\llcorner B}=P_{s}\left(2-P_{s}\right)-\sum_{m=1}^{M-1} P_{m}^{2}
$$

Where $\mathrm{P}_{\mathrm{m}}$ is the probability that the AWGN moves the transmitted MPSK symbol $\mathrm{m}$ decision regions away from the correct region and $\mathrm{P}_{\mathrm{s}}$ is the SER of the optimum MPSK detector in AWGN, whose decision rule is given as

$$
\underset{\theta_{k}}{\arg \max } \mathfrak{R}\left\{r_{k} e^{-j \theta_{k}}\right\}
$$

The lower bound in (20) is valid only when the received samples, $\left\{r_{k}\right\}$, are used to evaluate the MSDD's decision metric. However, the receiver proposed in section 3.1 uses the $\left\{\tilde{r}_{\mathrm{k}}\right\}$ to evaluate the decision metric given by (17). Therefore, the lower bound in (19) should be adjusted to account for the above fact. To this end, by replacing $\mathrm{r}_{\mathrm{k}}$ with $\tilde{\mathrm{r}}_{\mathrm{k}}$ in (20), one has that

$$
\arg \max _{\theta_{k}} \mathfrak{R}\left\{r_{k} e^{-j \theta_{k}}\right\}
$$

Eq. (21) denotes the decision rule of the optimum differential detector of MDPSK when $N=2$. The SER of this receiver is given by eq. (3)

$$
P_{s}=\frac{1}{\pi} \int_{0}^{\pi-\pi / m} \exp \left(\frac{-\gamma_{s} \sin ^{2}\left(\frac{\pi}{M}\right)}{1+\cos \left(\frac{\pi}{M}\right) \cos \theta}\right) d \theta
$$

We also use the distribution of the phase angle between two vectors corrupted by uncorrelated Gaussian noise to obtain $\mathrm{P}_{\mathrm{m}}$, after some manipulations, as

$$
\begin{gathered}
P_{m}=\frac{1}{2 \pi} \int_{0}^{\pi-\frac{(2 m-1) \pi}{M}} \exp \left(\frac{-\gamma_{s} \sin ^{2}\left(\frac{(2 m-1) \pi}{M}\right)}{1+\cos \left(\frac{(2 m+1) \pi}{M}\right) \cos \theta}\right) d \theta \\
-\frac{1}{2 \pi} \int_{0}^{\pi-\frac{(2 m+1) \pi}{M}} \exp \left(\frac{-\gamma_{s} \sin ^{2}\left(\frac{(2 m+1) \pi}{M}\right)}{1+\cos \left(\frac{(2 m+1) \pi}{M}\right) \cos \theta}\right) d \theta \\
\mathrm{m}=1,2, \ldots ., \mathrm{M}-1 .
\end{gathered}
$$

For the special case when $\mathrm{M}=2, P_{b}^{L B}$ can be expressed in closed-form as

$$
P_{b}^{L B}=e^{-\gamma_{b}}-\frac{1}{2} e^{-2 \gamma_{b}}
$$

Where $\gamma_{\mathrm{b}}$ denotes the SNR per bit. Note that for moderate to large values of SNR the square terms on the right of (19) are very small and can be ignored. Hence, for these SNR values $P_{b}^{L B}$ is approximately equal to $2 \mathrm{P}_{\mathrm{s}}$. 


\section{Numerical Results}

As mentioned in Section 2, the expression given in (4) is an approximation for the sampled received signal as it does not account for the amplitude attenuation caused by the frequency offset. It is, therefore, important to examine the receiver performance in the presence of this attenuation and indicate how good the aforementioned approximation is. To this end, we evaluate the error probability performance of the proposed MSDD receiver as a function of $\psi$ for $\gamma_{b}=10 \mathrm{~dB}$ and selected values of $\mathrm{N}$. We assume a rectangular pulseshaping filter is used at the transmitter. The results are shown in Fig.2 for binary DDPSK (BDDPSK) and quaternary DDPSK (QDDPSK) modulations. Note that the error probability performance at $\psi=0$ represents the case where eqs. (3) and (4) are identical. When $\psi<0.4$, the error probability is approximately constant for all examined values of $\mathrm{N}$. The error probability slightly increases when $\psi$ increases greater than 0.4 for both modulation schemes. However, the increase is small enough that we can consider eq. (4) a reasonable approximation for the sampled received signal.

The performances of ACD receiver for DDPSK signals and the proposed receiver with BDDPSK modulation for $\psi=0.3$ and selected values of $\mathrm{N}$ are depicted in fig. 3. It is assumed that the band pass filter prior to the ACD has a bandwidth of $8 \pi / T$. When $N=2$, the ACD outperforms the proposed receiver for $\mathrm{SNR} \geq 11 \mathrm{~dB}$. Increasing the observation interval improves the performance of the proposed receiver relative to the case where $\mathrm{N}=2$ rather rapidly. However, this increase does not have a significant impact on the performance of the ACD receiver. For example, when the average BER equals $10^{-6}$, increasing $\mathrm{N}$ from 2 to 8 results in a $3 \mathrm{~dB}$ SNR gain for the proposed receiver, whereas the corresponding gain for the ACD is approximately $0.35 \mathrm{~dB}$.

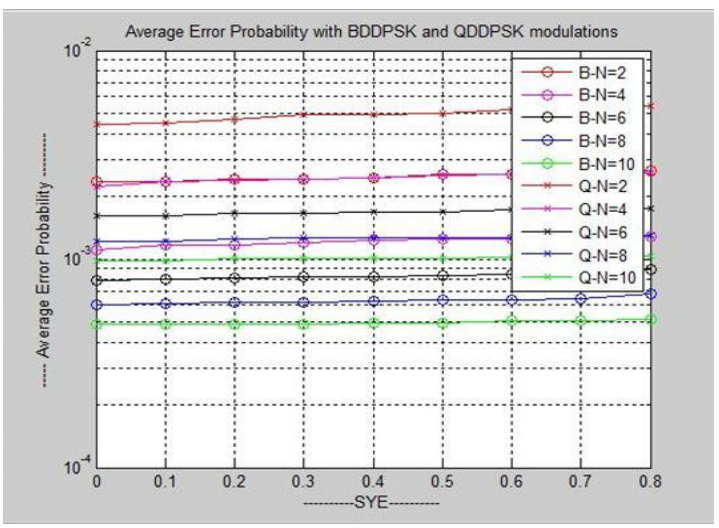

Fig. 2 The average error probability of the proposed for the

MSDD as a function of $\psi$ with BDDPSK \& QDDPSK modulation, modulation for $\mathrm{SNR}=10 \mathrm{~dB} \& \mathrm{~N}=2,4,6,8$ and 10 .

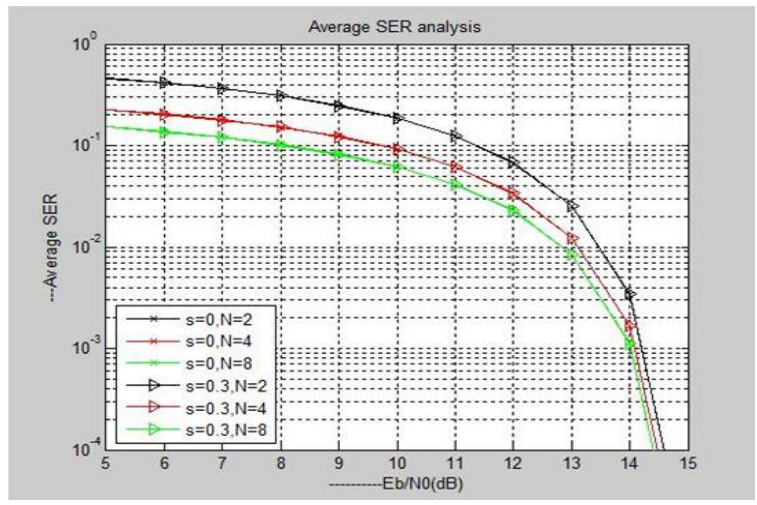

Fig. 4 The average SER as a function of SNR

Proposed MSDD receiver with QDDPSK $\psi=0.3 \&$ selected values of $\mathrm{N}$.

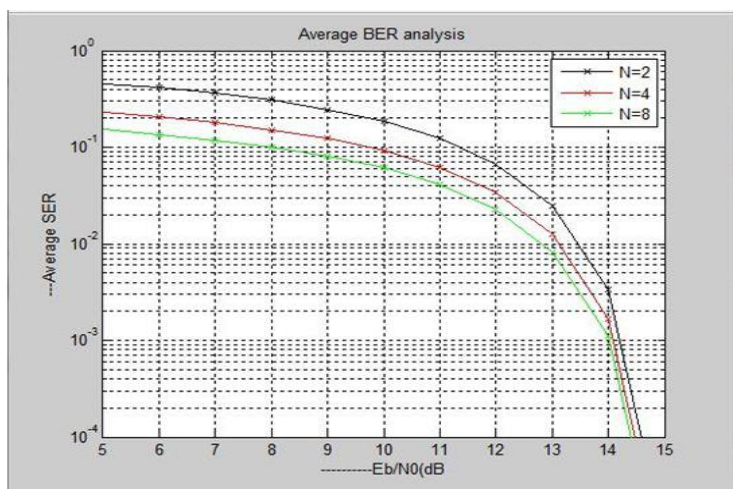

Fig.3 The average BER as a function of SNR for the proposed

proposed receiver and the autocorrelation demodulator detection

with BDDOSK modulation, $\psi=0.3$, and selected and 16

values of $\mathrm{N}$

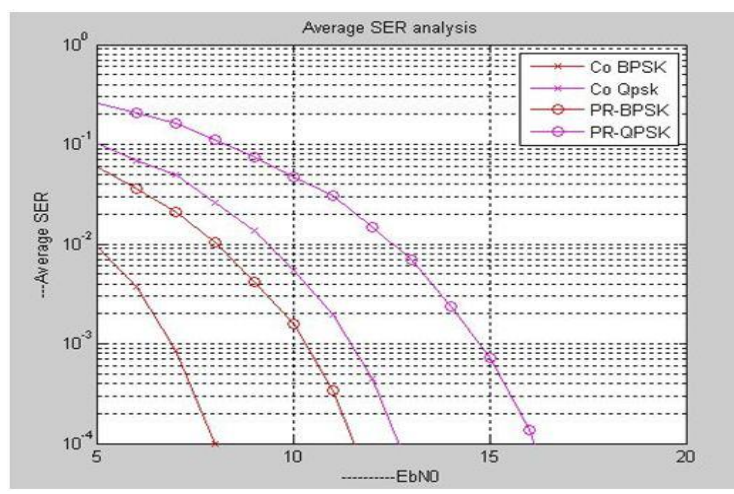

Fig.5 The lower bound on the BER of the receiver and the average BER for coherent of DPSK as a function of SNR for M=2,4,8 
Fig. 3 also shows that the lower bound becomes quite tight when $\mathrm{N}$ increases. Note that for $\mathrm{N}=16$ and $\mathrm{BER}=10^{-6}$, the proposed receiver achieves a gain of $2.5 \mathrm{~dB}$ relative to the ACD receiver. Fig 4 illustrates the error probability performance of the proposed receiver with QDDPSK modulation for the cases where $\psi=$ 0 and $\psi=0.3$, and selected values of $\mathrm{N}$. As seen in the figure, the performance of the receiver is approximately the same for both cases. Note that in this case, increasing the observation interval significantly improves the receiver performance. For example, when the $\mathrm{SER}=10^{-5}$, increasing $\mathrm{N}$ from 2 to 10 leads to a SNR gain of $3.5 \mathrm{~dB}$.

The ACD receiver suffers from a $3 \mathrm{~dB}$ SNR loss compared to the coherent detection of DPSK signals when $N \rightarrow \infty$ and SNR is very large. It is, therefore, desirable to investigate the SNR loss of the proposed receiver relative to the coherent detection of DPSK. Fig. 5 compares the lower bound on the BER of the proposed receiver with the BER of coherent demodulation of DPSK for $M=2,4,8$ and 16 . When the BER = $10^{-5}$ and BDDPSK modulation is used, the gap between the lower bound and coherent demodulation of DPSK is approximately $0.6 \mathrm{~dB}$. This gap increases to $2.5 \mathrm{~dB}$ for QDDPSK, which is still $0.6 \mathrm{~dB}$ less than that of the ACD-based MSDD.The gap approaches $3 \mathrm{~dB}$ for $\mathrm{M}=8$ and 16. However we still expect the proposed MSDD to outperform the ACD receiver. This is because the latter reaches the $3 \mathrm{~dB}$ gap when the AWGN is vanishingly small, whereas the proposed receiver does not require this extra condition.

\section{Conclusion}

Carrier frequency variation was shown to deteriorate the amplitude and the phase of a PSK signal transmitted over an AWGN channel. A MSDD scheme was proposed for detection of DDPSK signals in the presence of random frequency variation and AWGN. It was shown that the proposed receiver is insensitive to constellation rotation due to frequency offset, and is robust to amplitude attenuation provided that the frequency offset is not large. A lower bound on the error probability of the proposed receiver was derived. It was shown that the bound is quite tight for the case where the observation interval approaches infinity. It was also shown that the proposed receiver outperforms and ACD receiver when the number of received samples contributing to the detection process becomes large.

\section{Acknowledgement}

We thank our thesis advisors, Associate Professors J.Seetaram, G.Narsimhulu and P.Govardhan for their support and guidance. Their energy and insight at all levels of constant inspiration.

\section{References}

[1] J. G. Proakis, Digital Communications, 4th edition. McGraw-Hill, 2000.

[2] D.Divsalar and M.K.Simon, "Multiple-symbol differential detection of MPSK," IEEE Trans. Commun., vol. 38, no. 3, pp. 300308, Mar. 1990

[3] "Maximum-likelihood differential detection of uncoded and trellis coded amplitude phase moulation over AWGN and fading channels- metrics and performance," IEEE Trans. Commun., vol. 42, no. 1, pp. 76-89, Jan. 1994.

[4] S. G. Wilson, J. Freebersyser, and C. Marshall, "Multi-symbol detection of M-DPSK," in Proc. IEEE Global Telecommun. Conf., Nov. 1989, pp. 1692-1697.

[5] A. M. Rabiei and N. C. Beaulieu, "Multiple symbol differential detection of MPSK in the presence of frequency offset," in Proc. IEEE Int. Conf. Commun., vol. 1, May 2005, pp. 693-697.

[6] D. Divsalar and M. K. Simon, "Double differential detection," NASA New Technology Item 7170, Docket 17666, June 27; presented at IEEE Commun. Theory Workshop, Apr. 1987.

[7] Y. B. Okunev, V. A. Pisarev, and V. K. Reshemkin, "The design and noise-immunity of multiphase autocorrelation demodulators of second- order DPSK signals," Radiotekhnika, vol. 34, no. 6, pp. 60-63, 1979; Telecomm. Radio Eng., part 2, vol. 34, no. 6, 1979, pp. 60-63.

[8] Y. B. Okunev and L. M. Fink, "Noise immunity of various receiving methods for binary systems with second-order phase-difference mod- ulation," Radiotekhnika, vol. 39, no. 8, pp. 51-56, 1984; Telecommun. Radio Eng., vol. 39, no. 8, 1984, pp. 51-56.

[9] Y. B. Okunev and N. M. Sidorov, "Noise immunity of a carrier frequency-invarient demodulator of DPSK-2 signals," Radiotekhnika, no. 6, pp. 81-83, 1986; Telecommun. Radio Eng., no. 6, 1986, pp. 81-83.

[10] M. K. Simon and D. Divsalar, "On the implementation and performance of single and double differential detection schemes," IEEE Trans. Commun., vol. 40, no. 2, pp. 278-291, Feb. 1992.

[11] "Doppler-corrected differential detection of MPSK," IEEE Trans.Commun., vol. 37, no. 2, pp. 99-109, Feb. 1989.

[12] S. Niranjayan and N. C. Beaulieu, "On the integrated cross-noise term in correlation detectors," IEEE Trans. Commun., vol. 57, no. 11 , pp. 3244-3248, Nov. 2009.

[13] M. Pent, "Double differential PSK scheme in the presence of Doppler shift," in Proc.AGARD Conf. Digital Commun. Avionics, no. 239 , Nov. 1978 , pp. 43:1-43:11.

[14] M. K. Simon and M.-S. Alouini, Digital Communication over Fading Channels, 2nd edition. Wiley, 2005.

[15] R. F. Pawula, "A new formula for MDPSK symbol error probability," IEEE Commun. Lett., vol. 2, no. 10, pp. 271-272, Oct. 1998. 